\title{
Aspectos epidemiológicos dos óbitos por sepse neonatal no Estado da Bahia
}

\author{
Epidemiological aspects of deaths from neonatal sepsis in the State of Bahia
}

\begin{abstract}
Aspectos epidemiológicos de las muertes por sepsis neonatal en el Estado de Bahía
\end{abstract}
Kaique Vinicius da Cruz Santos Aguiar ${ }^{1 *}$, Greice Kely Oliveira de Souza1, Myllena Ferreira Rabelo, Jonas de Jesus Carvalho', Tiago de Freitas Sampaio', Janaína Magalhães Bispo Saba1', Kaluana Borges $^{1}$, Laís Santos Lima Oliveira ${ }^{1}$, Marcus Vinícius Mascarenhas Antunes¹.

\begin{abstract}
RESUMO
Objetivo: Identificar o perfil epidemiológico dos óbitos por sepse neonatal no estado da Bahia. Métodos: Estudo ecológico exploratório descritivo de série temporal, realizado através dos dados secundários do Sistema de Informação sobre Mortalidade (SIM). Foram estudados os óbitos por sepse neonatal ocorridos entre os anos de 2014 e 2018, entre indivíduos de 0 a 27 dias de nascido, residentes do Estado da Bahia. Baseou-se nas categorias da CID-10: A40, A41 e P36. Foram estudados varáveis neonatais, de óbito, da gestação, do parto e maternas. Resultados: Ocorreram 1114 óbitos neonatais por sepse, representando 9,4\% de todas as mortes entre faixa etária estudada no período, obtendo uma taxa de 1,09 óbitos por cada 1.000 nascidos vivos. Sendo caracterizados por bebês entre a faixa etária entre 0 a 6 dias de vida, recémnascido do sexo masculino, cor parda, aqueles com extremo baixo peso e prematuros de idade gestacional de 22 a 31 semanas. Houve domínio também entre aqueles que nasceram de gravidez unifetal, parto vaginal, de mães entre a faixa etária de 15 a 24 anos com escolaridade 8 a 11 anos. Conclusão: Os neonatos constituem um grupo mais vulnerável à sepse, aumentando o risco de mortalidade. Os resultados encontrados indicam necessidade de intensificação nas medidas de prevenção.
\end{abstract}

Palavras-chave: Recém-nascido, Sepse neonatal, Mortalidade, Perfil epidemiológico.

\begin{abstract}
Objective: To identify the epidemiological profile of deaths from neonatal sepsis in the state of Bahia. Methods: Exploratory ecological descriptive time series study, carried out using secondary data from the Mortality Information System (SIM). Deaths due to neonatal sepsis between 2014 and 2018 were studied among individuals aged 0 to 27 days born, residents of the State of Bahia. It was based on the ICD-10 categories: A40, A41 and P36. Neonatal, death, pregnancy, childbirth and maternal variables were studied. Results: There were 1114 neonatal deaths due to sepsis, representing $9.4 \%$ of all deaths among the age group studied in the period, with a rate of 1.09 deaths per 1,000 live births. Being characterized by babies between the age group between 0 to 6 days of life, newborn male, brown, those with extremely low weight and premature babies of gestational age of 22 to 31 weeks. There was also dominance among those who were born from single fetal pregnancy, vaginal delivery, from mothers between the age group of 15 to 24 years old with schooling from 8 to 11 years old. Conclusion: Neonates are a group more vulnerable to sepsis, increasing the risk of mortality. The results found indicate the need to intensify prevention measures.
\end{abstract}

Keywords: Newborn, Neonatal sepsis, Mortality, Epidemiological profile.

\section{RESUMEN}

Objetivo: Identificar el perfil epidemiológico de las muertes por sepsis neonatal en el estado de Bahía. Métodos: Estudio exploratorio ecológico descriptivo de series temporales, realizado con datos secundarios del Sistema de Información de Mortalidad (SIM). Se estudiaron las defunciones por sepsis neonatal entre 2014 y 2018, entre individuos de 0 a 27 días nacidos, residentes en el Estado de Bahía. Se basó en las categorías ICD-10: A40, A41 y P36. Se estudiaron variables neonatales, muerte, embarazo, parto y maternas. Resultados: Se registraron 1114 muertes neonatales por sepsis, lo que representa el $9,4 \%$ del total de

${ }^{1}$ Faculdade Pitágoras de Feira de Santana, Feira de Santana - BA. *E-mail: kaique.vinicius30@hotmail.com

SUBMETIDO EM: $3 / 2021$

ACEITO EM: 5/2021

PUBLICADO EM: 6/2021 
defunciones en el grupo de edad estudiado en el período, obteniendo una tasa de 1,09 defunciones por cada 1.000 nacidos vivos. Se caracteriza por bebés entre el grupo de edad entre 0 a 6 días de vida, recién nacidos varones, morenos, aquellos con peso extremadamente bajo y bebés prematuros de edad gestacional de 22 a 31 semanas. También hubo predominio entre los que nacieron de embarazo único fetal, parto vaginal, de madres entre el grupo de edad de 15 a 24 años con escolaridad de 8 a 11 años. Conclusión: Los recién nacidos son un grupo más vulnerable a la sepsis, aumentando el riesgo de mortalidad. Los resultados encontrados indican la necesidad de intensificar las medidas de prevención.

Palabras clave: Recién nacido, Sepsis neonatal, Mortalidad, Perfil epidemiológico.

\section{INTRODUÇÃO}

A incidência de sepse neonatal vem aumentando a cada ano, apesar do desenvolvimento na área médica, esse agravo é a principal causa de morbidade e mortalidade em recém-nascidos nos países em desenvolvimento, representando um problema de saúde pública relevante (HAMMAD E e ZAINAB MS, 2018). Tal condição pode ser decorrente de fatores de risco materno, transmitido verticalmente, intercorrências durante o parto, ou próprios do neonato (DORTAS AF, et al., 2019). O seu diagnóstico é difícil, uma vez que os sinais clínicos são inespecíficos e os exames complementares ainda têm baixa acurácia (PROCIANOY RS e SILVEIRA RC, 2020).

Cerca de $44 \%$ das mortes de crianças menores de cinco anos no mundo ocorrem durante o período neonatal. Considera-se que a sepse neonatal é um contribuinte significativo para a mortalidade neonatal mundialmente, visto que, cerca de 2,8 milhões de neonatos morrem a cada ano no mundo, onde em média $15 \%$ desses óbitos são causados por sepse (UNICEF, 2014). Nos Estados Unidos, cerca de 3,29\% dos recém-nascidos diagnosticados não sobrevivem (BOHANON FJ, et al., 2018). No Brasil em 2015, o agravo foi responsável por 1,69 mortes por cada 1.000 nascidos vivos, representando a quarta principal causa de mortes entre crianças menores de 5 anos no país (FRANÇA EB, et al., 2017).

A sepse neonatal compreende em uma doença sistêmica que se manifesta em recém-nascidos de até 28 dias de vida, é caracterizada por disfunção orgânica causada por uma infecção descontrolada, associado a alterações hemodinâmicas e outras manifestações clínicas graves (SHANE AL, et al., 2017). A sepse do recém-nascido é de choque frio, caracterizado por palidez, hipotermia, moteamento da pele, vasoconstriç̧ão periférica, oligúria e evidência de isquemia orgânica (MOREIRA MEL, et al., 2004).

O sistema imunológico imaturo do recém-nascido é um grande fator que contribui para o desenvolvimento da sepse neonatal (SINGH M, et al., 2020). Além disso, parto não supervisionado, asfixia ao nascer, baixo peso e necessidade de tecnologias utilizadas em unidade de terapia intensiva também são fatores de risco importantes associados a este agravo (OGUNDARE E, et al., 2019, SILVA SMR, et al., 2015).

A sepse neonatal é classificada como precoce ou tardia. Sepse neonatal precoce é quando as manifestações clínicas aparecem nas primeiras 48 a 72 horas de vida. A sepse de início precoce está relacionada a fatores pré-natais e do periparto, ou seja, são adquiridas antes ou durante o parto e geralmente representam transmissão vertical de mãe para filho. $E$ a sepse tardia é quando o quadro clínico se inicia após 72 horas de vida, está relacionada a fatores pós-natais atribuídos a organismos adquiridos de interação com o ambiente hospitalar ou a comunidade, na maioria das vezes relacionado a múltiplos procedimentos em unidades de terapia intensiva (UTIs) aos quais os recém-nascidos estão sujeitos (SILVEIRA RC e PROCIANOY RS, 2012, SHANE AL, et al., 2017).

Considerando a grande morbimortalidade por sepse entre bebês e os impactos da morte prematura de um indivíduo para família e sociedade, estudar o perfil da sepse neonatal é de grande relevância, visto que, através de estudos epidemiológicos recentes de um determinado local ou região, favorece para avaliação do sistema de saúde e subsídio de políticas públicas com estratégias bem-sucedidas para melhorar a sobrevida e reduzir a morbimortalidade neonatal daquela localidade. Dessa forma, o objetivo desse estudo foi identificar o perfil epidemiológico dos óbitos por sepse neonatal no estado da Bahia, ocorridos entre os anos 2014 a 2018. 


\section{MÉTODOS}

Estudo ecológico exploratório descritivo de série temporal, realizado através dos dados secundários do Sistema de Informação sobre Mortalidade (SIM). Foram estudados os óbitos por sepse neonatal ocorridos entre os anos de 2014 e 2018, entre indivíduos de 0 a 27 dias de nascido, residentes do Estado da Bahia, Brasil. Baseou-se nas categorias da Classificação Internacional de Doenças (CID-10): A40 (Septicemia estreptocócica), A41 (Outras septicemias) e P36 (Septicemia bacteriana do recém-nascido).

A Bahia é um estado do nordeste brasileiro possuindo em média 564 mil km² de área territorial e 14 milhões de habitantes. Os dados de saúde da Bahia utilizados para essa pesquisa foram coletados em 21 de outubro de 2020, através do Sistema de Informação sobre Mortalidade (SIM) via Departamento de Informática do Sistema Único de Saúde do Brasil (DATASUS). O SIM é um sistema de declaração de óbito informatizada, o qual foi criado pelo DATASUS para a obtenção regular de dados sobre mortalidade no país, através dele é possível a captação de dados sobre mortalidade, de forma abrangente, para subsidiar as diversas esferas de gestão na saúde pública (DATASUS, 2020).

As variáveis estudadas foram divididas e classificadas em três tipos, de acordo com suas características: 1- Variáveis neonatais e de óbito: ano do óbito, faixa etária, sexo, cor/raça, peso ao nascer; 2- Variáveis da gestação e do parto: tipo de gravidez, duração da gestação, tipo de parto; 3-Variáveis maternas: idade e escolaridade da mãe. Foi definido e bordado o tipo de morte neonatal classificada entre precoce ou tardia, considerou-se morte precoce para aqueles que tinham até seis dias de vida, e tardia, de sete a 27 dias de vida.

Além disso, foi calculado o coeficiente de mortalidade específica, através da divisão do número de óbitos por sepse neonatal, pela quantidade de nascidos vivos de mães residentes no mesmo local e período e multiplicou-se por 1.000. Para obtenção do número de nascidos vivos utilizou-se dados do Sistema de Informações sobre Nascidos Vivos (SINASC), fornecidos também pelo DATASUS. Como ferramenta de tabulação e análise descritiva dos dados utilizou-se o Software Microsoft Excel.

Por tratar-se de um estudo baseado em dados secundários fornecidos por um banco de dados de domínio público, não foi necessária aprovação de Comitê de Ética de Pesquisa.

\section{RESULTADOS}

No Estado da Bahia, entre os anos de 2014 a 2018, tiveram 11.775 óbitos entre neonatos ( 0 a 27 dias de vida), onde 1.114 desses óbitos foram causados por sepse, representando $9,4 \%$ das mortes da faixa etária no período. O coeficiente de mortalidade por sepse neonatal obteve taxa de 1,09 óbitos por cada 1.000 nascidos vivos, onde os anos de 2015 e 2016 obtiveram a maior incidência (1,21 e 1,16 óbitos por cada 1.000 nascidos vivos respectivamente) e a menor incidência foi em 2018 (0,84 mortes por cada 1.000 nascidos vivos). Além disso, observa-se que de 2015 para 2018 houve um declínio do quantitativo de mortes (Tabela 1).

Tabela 1 - Caracterização dos óbitos por sepse neonatal segundo ano do óbito e coeficiente de mortalidade especifica, $n=1.114$. Estado da Bahia - Brasil, 2014 - 2018.

\begin{tabular}{ccc}
\hline Ano do óbito & Óbitos $\left(\mathbf{n}^{\circ}\right)$ & $\begin{array}{c}\text { Mortalidade especifica ( } \mathbf{n}^{\circ} \text { de óbitos } \\
\text { por cada } \mathbf{1 . 0 0 0} \text { nascidos vivos) }\end{array}$ \\
\hline 2014 & 233 & 1,14 \\
2015 & 251 & 1,21 \\
2016 & 232 & 1,16 \\
2017 & 225 & 1,10 \\
2018 & 173 & 0,84 \\
\hline Total & 1114 & 1,09 \\
\hline
\end{tabular}

Fonte: Aguiar KVCS, et al., 2021; dados extraídos de DATASUS / SIM. 
Na distribuição etária, identificou-se que os recém-nascidos de 1 a 6 dias de vida obtiveram maior prevalência entre as mortes por sepse, responsável por quase metade do quantitativo total $44,7 \%(n=498)$, seguido daqueles com a faixa etária de 7 a 13 dias de vida dominante em $26,4 \%(n=294)$ dos óbitos. As mortes entre o período menor que 24 horas após o nascimento obteve $8,4 \%(n=94)$ (Tabela 2). Levando em consideração o período do óbito de acordo com a característica de óbito neonatal entre precoce ou tardio, pode observar o predomínio de óbitos precoce, aqueles ocorridos entre 0 a 6 dias de vida o qual representou $54 \%$.

$\mathrm{Na}$ variedade peso ao nascer, os neonatos nascidos com extremo baixo peso foi o grupo que mais morreu por sepse, visto que $29,3 \%(n=326)$ dos indivíduos mortos nasceram com 500 a 999 gramas e $8,4 \%(n=1,8)$ com menos de 500 gramas. Aqueles com peso ao nascer de 1000 a 1499 gramas, ou seja, recém-nascidos de muito baixo peso foi o segundo grupo mais prevalente entre os mortos por sepse no período estudado, obtendo $21,6 \%$ ( $n=241)$ do quantitativo de mortes, seguido dos que nasceram com 1500 a 2499 gramas, responsável por $19,6 \%(n=218)$ (Tabela 2$)$.

No que se refere ao sexo, o sexo masculino esteve maior incidência entre as mortes, onde obteve prevalente em 644 casos, representando um percentual de $57,8 \%$ e o sexo feminino $41,7 \%$ ( $n=465)$. Além disso, recém-nascidos da cor parda foram os que mais morreram responsável por $70,2 \%(n=782)$, seguido da cor branca $8,3 \%(n=92)$ e preta $2,3(n=23)$. Entretanto, cabe ressaltar que uma parte expressiva da variável raça/cor teve a informação ignorada, deixando $18,9 \%(n=210)$ registros sem essa informação, assim podendo representar um viés na análise dessa variável (Tabela 2).

Tabela 2 - Caracterização dos óbitos por sepse neonatal segundo faixa etária, sexo, cor/raça, peso ao nascer, $\mathrm{n}=1.114$. Estado da Bahia - Brasil, 2014 - 2018.

\begin{tabular}{|c|c|c|}
\hline Variável & $\mathbf{N}$ & $\%$ \\
\hline \multicolumn{3}{|l|}{ Faixa etária } \\
\hline Menos de 24 horas & 94 & 8,4 \\
\hline 1 a 6 dias & 498 & 44,7 \\
\hline 7 a 13 dias & 294 & 26,4 \\
\hline 14 a 20 dias & 142 & 12,7 \\
\hline 21 a 27 dias & 86 & 7,7 \\
\hline \multicolumn{3}{|l|}{ Sexo } \\
\hline Masculino & 644 & 57,8 \\
\hline Feminino & 465 & 41,7 \\
\hline Ignorado & 5 & 0,4 \\
\hline \multicolumn{3}{|l|}{ Cor/raça } \\
\hline Branca & 92 & 8,3 \\
\hline Preta & 26 & 2,3 \\
\hline Amarela & 1 & 0,1 \\
\hline Parda & 782 & 70,2 \\
\hline Indígena & 3 & 0,3 \\
\hline Ignorado & 210 & 18,9 \\
\hline \multicolumn{3}{|l|}{ Peso ao nascer } \\
\hline Menos de 500 gramas & 20 & 1,8 \\
\hline 500 a 999 gramas & 326 & 29,3 \\
\hline 1000 a 1499 gramas & 241 & 21,6 \\
\hline 1500 a 2499 gramas & 218 & 19,6 \\
\hline 2500 a 2999 gramas & 85 & 7,6 \\
\hline 3000 a 3999 gramas & 152 & 13,6 \\
\hline 4000 gramas ou mais & 9 & 0,8 \\
\hline Ignorado & 63 & 5,7 \\
\hline Total & 1114 & - \\
\hline
\end{tabular}

Fonte: Aguiar KVCS, et al., 2021; dados extraídos de DATASUS/ SIM. 
Quanto aos fatores relacionados a gestação, o tipo de gravidez que predominou entre as mães dos neonatos mortos por sepse foi a gravidez única (unifetal), o qual conteve $87,1 \%(n=970)$. A gravidez gemelar dupla esteve presente em 970 casos compreendendo $7,6 \%$, a gravidez gemelar tripla ou mais $0,4 \%$, porém em $4,8 \%$ dos registros tiveram essa informação ignorada (Tabela 3).

Os prematuros que tinham idade gestacional de 22 a 27 semanas e 28 a 31 semanas também obtiveram dominância entre os óbitos, responsáveis por $22,4 \%(n=249)$ e $21,9 \%(n=244)$ consecutivamente. Além disso, verificou-se que neonatos com idade gestacional de 37 a 41 semanas representou $19,4 \%(n=216)$ entre os indivíduos mortos por sepse e os que nasceram com idade gestacional de 32 a 36 semanas obteve $15,9 \%$ ( $n=177)$, no entanto a variável teve a informação ignorada em $16,2 \%$ dos registros. $E$ se tratando do tipo de parto, houve o predomínio do parto vaginal possuindo prevalente em $57,4 \%(n=639)$ dos casos, o parto cesáreo correspondeu 37,9\% $(n=422)$ (Tabela 3).

Tabela 3 - Caracterização dos óbitos por sepse neonatal segundo o tipo de gravidez, duração da gestação, tipo de parto, $\mathrm{n}=1.114$. Estado da Bahia - Brasil, 2014 - 2018.

\begin{tabular}{ccc}
\hline Variável & N & $\%$ \\
\hline Tipo de gravidez & & 87,1 \\
Única & 970 & 7,6 \\
Dupla & 85 & 0,4 \\
Tripla e mais & 54 & 4,8 \\
lgnorada & & \\
\hline Duração da gestação & 41 & 3,7 \\
\hline Menos de 22 semanas & 249 & 22,4 \\
22 a 27 semanas & 244 & 21,9 \\
28 a 31 semanas & 177 & 15,9 \\
32 a 36 semanas & 216 & 19,4 \\
37 a 41 semanas & 7 & 0,6 \\
42 semanas e mais & 180 & 16,2 \\
lgnorado & & 57,4 \\
\hline Tipo de parto & 639 & 37,9 \\
\hline Vaginal & 422 & 4,8 \\
Cesáreo & 53 & - \\
lgnorado & $\mathbf{1 1 1 4}$ & \\
\hline Total & & \\
\hline
\end{tabular}

Fonte: Aguiar KVCS, et al., 2021; dados extraídos de DATASUS / SIM.

Com relação à idade das mães dos recém-nascidos estudados, esteve em maior prevalência a faixa etária de 20 a 24 anos e 15 a 19 anos, responsáveis pelo quantitativo de $21,9 \%(n=244)$ e $21 \%(n=234)$ consecutivamente. Em seguida as mães com idade entre 25 a 29 anos, onde obteve 18,4\% $(n=205)$, aquelas com a faixa etária entre 35 a 39 anos representou $11,4 \%(n=127)$. Apesar de não obter dominância entre os casos chama atenção do quantitativo de mães na fase inicial da adolescência, ou seja, aquelas entre 10 a 14 anos que representou 2,2\% $(n=24)$ (Tabela 4).

$\mathrm{Na}$ análise da escolaridade da mãe, observou-se a maior incidência entre o grupo de 8 a 11 anos de estudo, o qual obteve um percentual de $40,8 \%(n=455)$, em seguida aquelas tinham entre 4 a 7 anos de estudo representou $22,2 \%(n=247)$. Em menor percentual esteve as que estudaram de 12 anos e mais $(7,9 \%), 1$ a 3 anos (6,3\%), nenhuma escolaridade (3,0\%) (Tabela 4$)$. 
Tabela 4 - Caracterização dos óbitos por sepse neonatal segundo a idade da mãe e escolaridade da mãe, $\mathrm{n}=1.114$. Estado da Bahia - Brasil, 2014 - 2018.

\begin{tabular}{|c|c|c|}
\hline Variável & $\mathbf{N}$ & $\%$ \\
\hline \multicolumn{3}{|l|}{ Idade da mãe } \\
\hline 10 a 14 anos & 24 & 2,2 \\
\hline 15 a 19 anos & 234 & 21,0 \\
\hline 20 a 24 anos & 244 & 21,9 \\
\hline 25 a 29 anos & 205 & 18,4 \\
\hline 30 a 34 anos & 177 & 15,9 \\
\hline 35 a 39 anos & 127 & 11,4 \\
\hline 40 a 44 anos & 27 & 2,4 \\
\hline 45 a 49 anos & 2 & 0,2 \\
\hline Idade ignorada & 74 & 6,6 \\
\hline \multicolumn{3}{|l|}{ Escolaridade mãe } \\
\hline Nenhuma & 33 & 3,0 \\
\hline 1 a 3 anos & 70 & 6,3 \\
\hline 4 a 7 anos & 247 & 22,2 \\
\hline 8 a 11 anos & 455 & 40,8 \\
\hline 12 anos e mais & 88 & 7,9 \\
\hline Ignorado & 221 & 19,8 \\
\hline Total & 1114 & - \\
\hline
\end{tabular}

Fonte: Aguiar KVCS, et al., 2021; dados extraídos de DATASUS / SIM.

\section{DISCUSSÃO}

O estudo evidenciou que entre 2014 e 2018 no estado da Bahia ocorreu 1.114 óbitos por sepse neonatal, representando $9,4 \%$ de todas as mortes entre indivíduos da faixa etária de 0 a 27 dias de vida. 0 coeficiente de mortalidade no período obteve uma taxa de 1,09 óbitos por cada 1.000 nascidos vivos, achado semelhante ao estudo de Benincá VM, et al. (2013) que constatou uma taxa de 1,08 óbitos por 1.000 nascidos vivos na macrorregião Sul de Santa Catarina no período de 1996 a 2009. Já Alves JB, et al. (2018) que pesquisou os óbitos por sepse neonatal em um município do Sul do Brasil, evidenciou uma taxa de 2,3 por 1.000 nascidos vivos. Segundo a revisão sistemática de Heath PT e Jardine LA (2014), a incidência geral de sepse neonatal varia de 1,0 a 8,0 bebês por 1.000 nascidos vivos em países desenvolvidos.

Quanto as variáveis neonatais, os óbitos foram caracterizados por bebês entre a faixa etária de 0 a 6 dias de vida (classificado como óbito neonatal precoce), recém-nascido do sexo masculino, cor parda, aqueles com extremo baixo peso (menos de 500 gramas a 999 gramas) e prematuros de idade gestacional de 22 a 31 semanas.

Contribuindo com os achados desse estudo, a pesquisa de Meireles LA, et al. (2011) também evidenciou entre as mortes neonatais por sepse a prevalência entre bebês do sexo masculino, nascidos com $\leq 1.500$ gramas e aqueles classificados como pequeno para idade gestacional. Tanto o baixo peso ao nascer quanto a prematuridade também apresentaram maiores percentuais das mortes por sepse neonatal no estudo de Alves JB, et al. (2018). Em relação ao período de ocorrência do óbito, nesse mesmo estudo houve predomínio de mortes após o sexto dia de vida da criança, ou seja, óbito neonatal tardio.

Em um estudo de coorte realizado nos Estados Unidos, o qual incluiu 160.677 neonatos sépticos, nem a raça e nem o gênero afetaram adversamente as taxas de mortalidade, entretanto negros tiveram menor tempo de permanência em comparação com brancos. Além disso, os bebês nascidos com menos de 1.500 gramas aumentaram significativamente as chances de morte em comparação com recém-nascidos de peso normal (> 2.500 gramas) (BOHANON FJ, et al., 2018). No estudo de Jiang S, et al. (2020) realizado na China, a taxa 
de letalidade de sepse neonatal foi a mais alta entre bebês com idade gestacional $<26$ semanas $(44,1 \%)$ e constatou 64,6\% de prevalência de sepse tardia. Na revisão sistemática de Yusef D, et al. (2018), constatou maior frequência nas evidências estudadas de sepse de início tardio, com maior prevalência entre a faixa etária média de 7 dias de vida, prematuros, neonatos idade gestacional mediana de 31 semanas e peso médio ao nascer de $1,6 \mathrm{~kg}$.

Ainda se tratando do estudo de Bohanon FJ, et al. (2018), foi evidenciado várias disparidades sociodemográficas que aumentaram significativamente a mortalidade. Pacientes que não tinham seguro privado de saúde aumentaram a mortalidade, além disso, a baixa renda familiar aumentou as chances de morte em comparação com as famílias mais ricas. Logo, levando compreender que os fatores sociodemográficos é um importante determinante a ser avaliado, entretanto, no Brasil não há muitas evidencias a respeito.

Essa pesquisa também evidenciou que as mortes por sepse em recém-nascidos tiveram dominância entre aqueles que nasceram de gravidez unifetal, parto vaginal, de mães entre a faixa etária de 15 a 24 anos com escolaridade 8 a 11 anos, como na pesquisa de Alves JB, et al. (2018), que obteve maior frequência de mortes por sepse entre neonatos provenientes também de gestação única, por parto vaginal, entre filhos de mães com menos de 35 anos, recém-nascidos de mães primigestas, com escolaridade de oito ou mais anos de estudo. Alves JB, et al. (2018) também estudou às intercorrências na gestação relacionadas, onde obteve destaque pré-eclâmpsia, infecção de trato urinário na gestação e trabalho de parto prematuro.

Conforme Oliveira COP, et al. (2016) os fatores de risco para o desenvolvimento da sepse neonatal estão relacionados a três condições: fatores gestacionais e maternos; condições de nascimento e prematuridade; e fatores relacionados ao ambiente da Unidade de Terapia Intensiva Neonatal (UTIN). A prematuridade e o baixo peso ao nascer prevaleceram em todos os estudos como fator preditivo de maior evidência. A idade gestacional, infecção do trato geniturinário materno e ruptura prematura das membranas amnióticas são os principais fatores de risco gestacionais intervenientes na sepse neonatal. Os fatores relacionados ao ambiente da UTIN contemplam o tempo médio de permanência hospitalar, uso de dispositivos invasivos, tais como o cateter PICC, ventilação mecânica invasiva e nutrição parenteral, que são agravados pela imaturidade imunológica do recém-nascido. Dortas ARF, et al. (2019) ainda acrescenta como fatores de risco importante: o apgar baixo, ruptura prolongada de membranas amnióticas, deficiência de G6PD, pré-natal inadequado e hospitalização prévia.

Entre os fatores de risco para a sepse precoce, envolvidos principalmente com fatores maternos ou da gestação, destacam-se: ruptura prematura ou prolongada de membranas, corioamnionite, infecção materna do trato urinário, colonização materna por Streptococcus beta hemolítico do grupo B, parto prematuro, febre materna, líquido amniótico fétido e alterações laboratoriais (leucocitose) durante o pré-natal. E a sepse tardia está associada a fatores pós-parto e a numerosos tratamentos na unidade de terapia intensiva neonatal aos quais os recém-nascidos são suscetíveis, como dispositivos e procedimentos invasivos, nutrição parenteral, antibioticoterapia de amplo espectro, prematuridade e permanência hospitalar prolongada, intervenções cirúrgicas, transmissão horizontal, violando os princípios de prevenção e controle de infecções hospitalares, como higiene das mãos, desinfecção inadequada do ambiente e superpopulação de recém-nascidos na enfermaria neonatal (SANTOS ZMA, et al., 2020).

Nesse estudo não foi possível avaliar os agentes etiológicos mais envolvidos com o agravo, entretanto, a pesquisa de Lopes GK, et al. (2008), realizada no Hospital Universitário de Londrina, as bactérias mais comumente isoladas em neonatos com sepse foram Staphylococcus coagulase negativa (34,9\%), Escherichia coli $(13,8 \%)$ e Klebsiella pneumoniae (13,5\%), e quanto à sensibilidade aos antimicrobianos, $46,7 \%$ das bactérias isolados foram do tipo multirresistentes $(77,2 \%)$. Dados semelhantes com o estudo de Meireles LA, et al. (2011), realizado no Hospital Universitário Antônio Pedro, onde evidenciou a Klebsiella pneumoniae, o Staphylococcus coagulase negativa e o S. aureus como os microorganismos mais envolvidos entre os casos de sepse neonatal, onde bactérias classificadas como multirresistentes também mostrou quantitativo relevante entre os casos estudados.

Com os resultados desse estudo indicam a necessidade de intensificação nas medidas de prevenção da sepse neonatal. As medidas de prevenção e controle das infecções no período neonatal representam um 
desafio para os profissionais envolvidos na assistência, principalmente em unidades de terapia intensiva neonatal, uma vez que os recém-nascidos são submetidos a muitos procedimentos invasivos durante a internação hospitalar, aumentando o risco de aquisição de infecções neste período (TOMAZ VS, et al., 2011).

Foi possível observar nesse estudo que o desfecho ignorado obteve um quantitativo relevante entre as variáveis estudadas, isso por consequências de erro do sistema de notificações em saúde ou déficit no preenchimento dos atestados de óbitos pelos profissionais. Esse fator pode interferir na análise dos dados da pesquisa. Além disso, o SIM não fornece outras informações relevantes, como quando as manifestações clínicas começaram para análise das tendências de sepse neonatal precoce ou tardia, frequência de prénatal, agente etiológico envolvido e variáveis sociodemográficos relacionadas. Com isso, é sugerido novos estudos que possam ampliar as tendências.

\section{CONCLUSÃO}

Os neonatos constituem um grupo mais vulnerável à sepse. O estudo identificou altas taxas de mortalidade por sepse no período neonatal no estado da Bahia, caracterizados por óbito neonatal precoce, recém-nascido do sexo masculino, cor parda, aqueles com extremo baixo peso, prematuros classificados como pequeno para idade gestacional, nascidos de gravidez unifetal, parto vaginal, de mães entre a faixa etária de 15 a 24 anos com escolaridade 8 a 11 anos. O conhecimento desses fatores possibilita identificação de fatores de risco permitindo uma melhor abordagem aos recém-nascidos vulneráveis e subsídio de políticas públicas e estratégias de prevenção para melhorar a sobrevida e reduzir a morbimortalidade neonatal. Os resultados encontrados indicam necessidade de intensificação nas medidas de prevenção.

\section{REFERÊNCIAS}

1. ALVES JB, et al. Sepse neonatal: mortalidade em município do sul do brasil, 2000 a 2013. Revista Paulista de Pediatria, 2018; 36(2): $132-140$.

2. BENINCÁ VM, et al. Perfil epidemiológico dos óbitos por sepse neonatal na macrorregião Sul de saúde catarinense no período de 1996 a 2009. Arquivos Catarinenses de Medicina, 2013; 42(2), 20-26.

3. BOHANON FJ, et al. Race, Income and Insurance Status Affect Neonatal Sepsis Mortality and Healthcare Resource Utilization. The Pediatric Infectious Disease Journal, 2018; 37(7): 1-20.

4. DATASUS. 2020. In: Sistema de Informação sobre Mortalidade (SIM). Brasília: Ministério da Saúde. Disponível em:http://www2.datasus.gov.br/DATASUS/index.php?area=02. Acesso em: 21 de outubro de 2020.

5. DORTAS AF, et al. Fatores de risco associados a sepse neonatal: artigo de revisão. Revista Eletrônica Acervo Científico, 2019; 7: 18.

6. DORTAS ARF, et al. Fatores de risco associados a sepse neonatal: artigo de revisão. Revista Eletrônica Acervo Científico, $2019 ; 7$ : 1-8.

7. FRANCA EB, et al. Principais causas da mortalidade na infância no Brasil, em 1990 e 2015: estimativas do estudo de carga global de doença. Revista Brasileira de Epidemiologia, 2017; 20(1): 46-60.

8. HAMMAD E, ZAINAB MS. Meta-Analysis on Factors Influencing Early Onset Neonatal Sepsis. Scholar Journal of Applied Sciences and Research, 2018; 1(8): 20-22.

9. HEATH PT, JARDINE LA. Neonatal infections: group B streptococcus. BMJ Clinical Evidence, 2014; 02(323): 1-9.

10. JIANG S, et al. Epidemiology and microbiology of late-onset sepsis among preterm infants in China, 2015-2018: a cohort study. International Journal Of Infectious Diseases, 2020; 96: 1-9.

11. LOPES GK, et al. Estudo epidemiológico das infecções neonatais no Hospital Universitário de Londrina, Estado do Paraná. Acta Scientiarum. Health Science, 2008; 30(1): 55-63.

12. MEIRELES LA, et al. Avaliação do diagnóstico da sepse neonatal: uso de parâmetros laboratoriais e clínicos como fatores diagnósticos. Rev. Esc. Enferm, 2011; 45(1): 33-39.

13. MOREIRA MEL, et al. O recém-nascido de alto risco: teoria e prática do cuidar. Rio de Janeiro: Editora Fiocruz, 2004; 265p.

14. OGUNDARE E, et al. Presentation and outcomes of early and late onset neonatal sepsis in a Nigerian Hospital. African Health Sciences, 2019; 19(3): 2390-2399.

15. OLIVEIRA COP, et al. Fatores de risco para sepse neonatal em unidade de terapia: estudo de evidência. Cogitare Enfermagem, 2016; 21(2): 01-09.

16. PROCIANOY RS, SILVEIRA RC. The challenges of neonatal sepsis management. Jornal de Pediatria, 2020; 96: 80-86.

17. SANTOS ZMA, et al. Sepse neonatal, avaliação do impacto: uma revisão integrativa. Bionorte, 2020; $9(1): 47-58$. SHANE AL, et al. Neonatal sepsis. The Lancet, 2017; 390(10104): 1770-1780.

19. SILVA SMR, et al. Late-onset neonatal sepsis in preterm infants with birth weight under $1.500 \mathrm{~g}$. Revista Gaúcha de Enfermagem, 2015; 36(4): 84-89.

20. SILVEIRA RC, PROCIANOY RS. Uma revisão atual sobre sepse neonatal. Boletim Científico de Pediatria, 2012; 1(1): 29-35.

21. SINGH M, et al. Neonatal Sepsis. In: StatPearls, 2020. Disponível em: https://www.ncbi.nlm.nih.gov/books/NBK531478/. Acesso em: 8 de dezembro de 2020.

22. TOMAZ VS, et al. Medidas de prevenção e controle de infecções neonatais: opinião da equipe de enfermagem. Rev Rene, 2011; 12(2): 271-278.

23. UNICEF. Níveis tendências da mortalidade em: https://www.unicef.org/media/files/Levels_and_Trends in_Child_Mortality_2014.pdf. Acessado em: 19 de dezembro de 2020.

24. YUSEF D, et al. Clinical characteristics and epidemiology of sepsis in the neonatal intensive care unit in the era of multi-drug resistant organisms: a retrospective review. Pediatrics \& Neonatology, 2018; 59(1): 35-41. 